\title{
MINIATURIZING THE MECHANICAL RELAY FOR SIGNAL AND POWER CONTROL
}

\author{
Chris Keimel, Marco Aimi, Oliver Boomhower, Alex Corwin and Andrew Detor \\ GE Global Research, Niskayuna, New York USA
}

\begin{abstract}
Traditionally, most Micro ElectroMechanical Systems (MEMS) have been made using silicon as the mechanical material; we have developed a metal based MEMS device technology that miniaturizes the basic DC, AC and RF relay. Advances in MEMS switches have primarily focused on RF switches [1], with power levels typically in the 10s to $100 \mathrm{~W}$ [2] and contact related failure mechanisms [3,4]. We have developed an electrostatically actuated MEMS relays, has been developed (Fig. 1) and is capable of switching in $~ 3$ usec, sustaining more than $400 \mathrm{~V}$ across its open contacts and controlling loads of 100 s of watts to a few kilowatts.
\end{abstract}

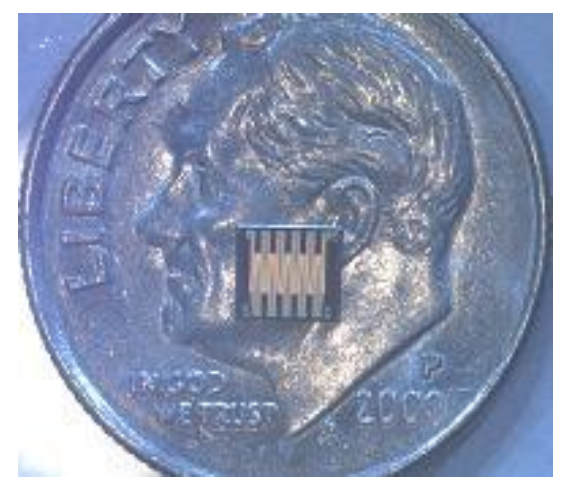

Figure 1: A Ni-alloy relay containing 400 switches capable of switching kilowatts of power.

\section{INTRODUCTION}

Mechanical relays used to switch electrical current or signals are not a new technology, being first invented by Joseph Henry in the 1830 s to improve the electric telegraph. Mechanical relays have seen significant improvements in their materials, design, and performance in the following century. Mechanical relays provide a highly conductive Ohmic pathway when closed and the isolation of an air gap when open. The invention of the transistor in 1947 provided the foundation for the solid state relay that was a smaller, faster, and lower power consumption device than the mechanical relay. However, solid state devices lacked the isolation of an air gap and the low on state losses of an Ohmic contact. With the development of MEMS in the 1970s came the opportunity to miniaturize the mechanical relay while also giving it the speed and low power operation of solid state devices. In recent decades MEMS relay technology has, and is continuing to be, developed for high performance RF switching applications.

Newly engineered micromechanical structural materials are required to go beyond the signal transmission and sensing capabilities of present silicon based MEMS sensors. To manipulate significant power at the micro scale requires materials with the mechanical properties near that of silicon along with the conductivity of metals. We have developed and characterized a nanostructured $\mathrm{Ni}$-alloy electroplating process that has the conductivity of nickel, a tensile strength $>1 \mathrm{GPa}$ and demonstrated the alloy's process integration capability by

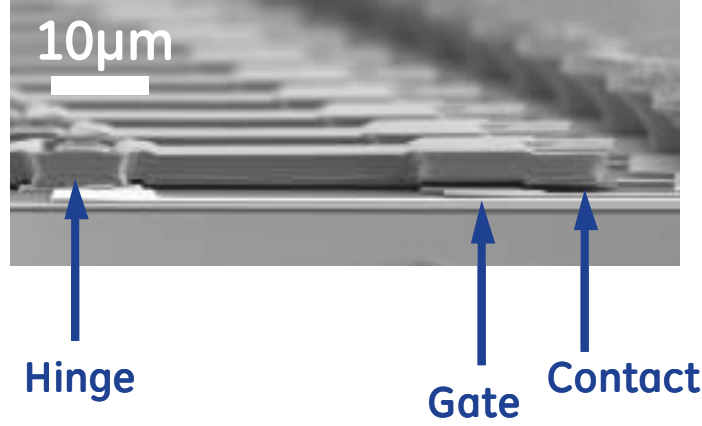

Figure 2: A cross sectional SEM view showing the beam, gate and contact of an individual MEMS relay in a switch array.

fabricating arrays of switches (figure 2) capable of transmitting and switching more than 1 kilowatts of power. The minimal strain rate of the nanostructured Ni-alloy makes possible cantilever switches with decades of operation at elevated temperatures. We have characterized the time-dependent mechanical behavior of the nickel alloy in comparison to other materials and through micro-tensile measurements extracted validated materials parameters, such as activation energy and activation volume, and used those parameters in a lifing model to predict operational life of the actuator.

In addition, hundreds of volts can be sustained across micro scale contacts separated by just a 1 micron gap. This sustained voltage of more than $300 \mathrm{~V}$ is possible due to the gap being smaller than the mean free path of the gas molecules and thus minimizing the effect of avalanche breakdown by minimizing the ionization potential [5]. At gaps smaller than the Paschen minimum of $327 \mathrm{~V}$ in air, field emission becomes significant. The contacts and their roughness have been designed and fabricated to minimize field emission effects at small gaps and have enabled sustained voltages $>300 \mathrm{~V}$ across a 1 um gap, a value that exceeds the perceived limits of the modified Paschen curve.

\section{RESULTS}

Silicon's mechanical properties have established MEMS as a technology for sensors and actuators and the numerous devices on the market today are a testament to its success. To move beyond sensors and actuators requires a micro fabrication compatible material set that provides added functionality by combining the mechanical properties of silicon with the electrical properties of a conductor. Enabling actuators to efficiently conduct allows them to now transmit, without significant joule heating, substantially more power than if they were composed of silicon. Actuators that can transmit and control signals and power have the capability to enable a new class of micro scale devices that move beyond sensing energy to now being able to manage and manipulate electrical signals and energy.

Solid-State Sensors, Actuators and Microsystems Workshop Hilton Head Island, South Carolina, June 8-12, 2014 
The inherent material challenge in enabling an Ohmic MEMS switch is that pure metals are great conductors, however their low yield stress (10s to $100 \mathrm{~s} \mathrm{MPa}$ ) limits the actuator design space and their grain structure instability under temperature limits device reproducibility and reliability. Resistance to permanent creep deformation during operation is a requirement for any cantilever style MEMS switch. If the cantilever beam material cannot withstand operating stresses during switching cycles, the contact gap in the 'off' state can decrease to such an extent that the switch itself no longer functions (i.e. it remains 'on' even without an applied gate voltage). Figure 3 demonstrates how contact gap in the 'off' state can change over time at several different temperatures during operation of a pure gold cantilever beam MEMS device. It is important to note that the time in figure 3 refers to total 'on' time of the switch where the beam experiences higher stresses that drive creep deformation. Gold cantilever switches, operated at $\sim 100 \mathrm{MPa}$ stress, cycled at a $50 \%$ duty cycle between $40 \mathrm{C}$ and $100 \mathrm{C}$ showed a downward deflection (of the open state) in the beams.

A Ni-alloy based electroplating conditions and MEMS based process integration were developed to produce cantilevered micro actuators that have the combined mechanical properties near that of silicon with the conductivity of a metal. The electroplated Ni-alloy has a yield strength $>1 \mathrm{GPa}$ and a resistivity around $7 \times 10^{-6} \mathrm{Ohm}-\mathrm{cm}$. The alloy's nanocrystalline grain structure $(<10 \mathrm{~nm})$ has been extensively characterized through TEM analysis and its composition engineered to maintain both grain stability and a minimal strain rate, for typical MEMS loads, up to $500{ }^{\circ} \mathrm{C}$ under a variable load as shown in figure 4. To evaluate the creep behavior of MEMS cantilever beam materials in the microtensile tester displacement is fixed and load is measured over time. In one test it is possible to back out strain rate over a range of stresses. Another advantage of the stress relaxation test is that accurate strain measurement in the gage section of the specimen is not needed.

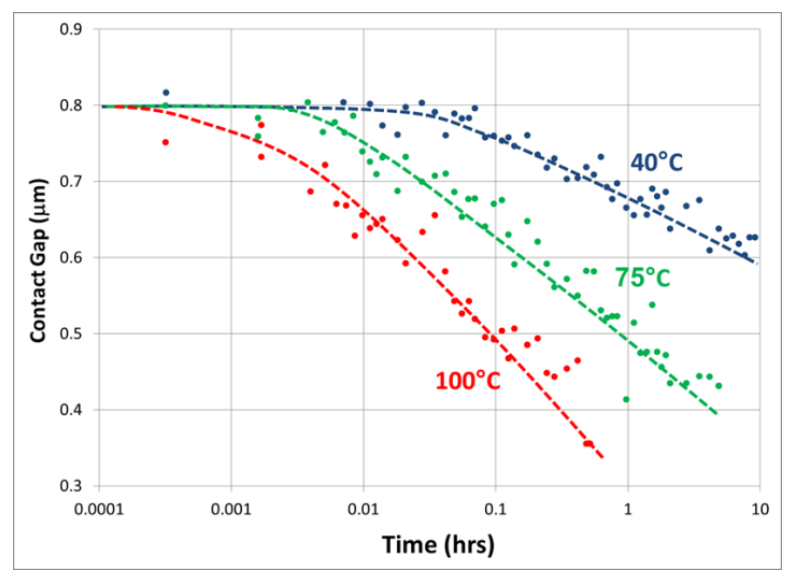

Figure 3: MEMS switch contact gap in the 'off' state as a function of 'on' time, demonstrating how the gap can decrease over time due to creep of a pure gold cantilever beam material. Increasing temperature promotes more rapid creep deformation.
With small specimen geometry and using resistance heating, the stress relaxation test only requires a constant fixed displacement, which the machine is capable of, and accurate load measurement from the load cell. Displacement is rapidly increased to a prescribed starting load, and then held fixed. Load drop is then measured as a function of time and temperature. The results of the stress relaxation tests determine a strain rate profile and enable the determination of the material specific parameters used to model time-dependent deformation of a suspended microstructure under load.

The plot in figure 5 depicts the modeled and expected deformation of three different materials, gold, a gold alloy and a nanocrystalline nickel alloy, under a typical peak stress of 120 $\mathrm{MPa}$ at the anchored hinge point when the system is isothermal at $85{ }^{\circ} \mathrm{C}$. The displacement model shows that the pure $\mathrm{Au}$ cantilever deflects $20 \%$ of its original $1 \mu \mathrm{m}$ gap in only 4 days while it takes more than 2 decades for the nanocrystalline $\mathrm{Ni}$ alloy cantilevers to deflect $20 \%$ under the identical load conditions.

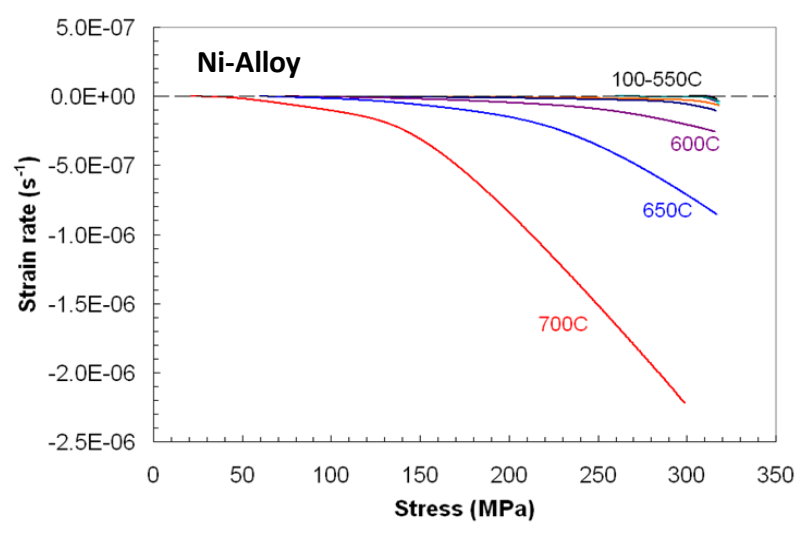

Figure 4: Strain rate as a function of applied stress derived from the raw load-time data. The data in this plot are directly used to calibrate a creep model for the material as explained in the text.

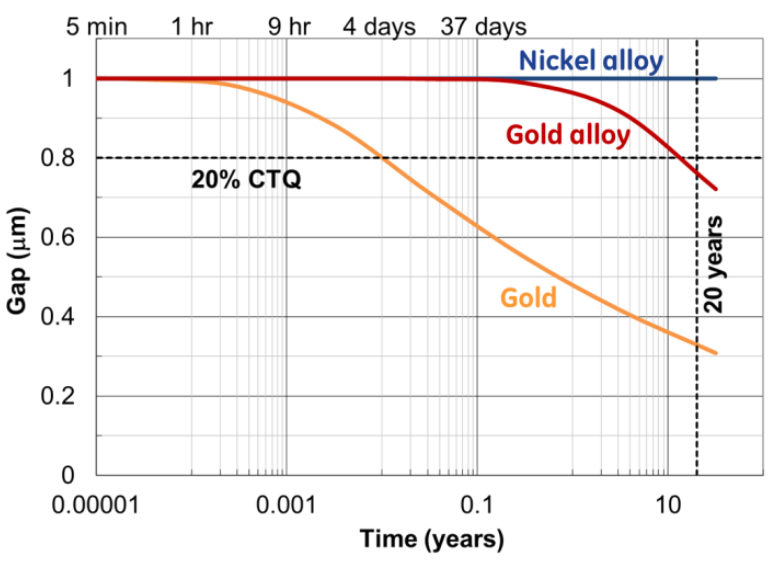

Figure 5: Predicted gap change as a function of time for three different beam materials. This prediction uses the model outlined in using the material parameters from microtensile testing described in previous plot. 
Grain size and uniformity were controlled during the electroplating process to eliminate cantilever warp and allow multiple microns of deposition with a very smooth surface finish. Unlike polysilicon, the deposition is done near room temperature which allows the formation of the structural elements to take place at any point during a device process flow, even after metal deposition. Released cantilevers and other thin film electroplated test structures were used in accelerated life tests to experimentally validate decade long projections. Both experimental and model results indicate that the nanocrystalline material sets exhibit reduced stress induced deformation that limits device performance.

In addition to a structurally stable metal beam, the ability to withstand hundreds of volts across micro and sub-micro gaps is required for relays to switch and control power. The standoff voltage is the maximum voltage that can appear between the substrate contact and the beam before electrical breakdown occurs across the air gap. This failure mode can be observed through two mechanisms. The first is the traditional electrical breakdown / arcing between two conductors across a gap, while the second is based on self-actuation of the MEMS switch. Similar to how a voltage between the gate and beam provides an electrostatic force to moves the beam, a voltage between the contact and the beam will also generate an electrostatic force. As the voltage between the beam and contact increases the beam begins to deflect towards the contact. As this voltage increases further, the beam goes beyond the instability point and snaps into the contact causing a self-actuation failure. This failure mode can be mitigated by altering the stiffness of the beam, beam to contact gap, and the nominal area of the contact and bump. Of these variables, only the areas of the bump and contact can be changed without affecting other aspects of the operation of the switch, such as gate voltage. Both simulation and experiments were run to determine the impact of changing these feature sizes. The results (Figure 6) show that as the contact electrode overlap area is decreased, the standoff voltage increases, up to a point where the electrical breakdown occurs at approximately $350 \mathrm{~V}$ as shown in blue. The pink data points are simulated breakdown voltages due to self-actuation limit of the switch. There is good agreement between the experiment and simulation below $350 \mathrm{~V}$, but experiments showed an upper limit to the max voltage despite reduced contact size. The observed experimental limit coincided with the minimum of the Paschen curve [6].

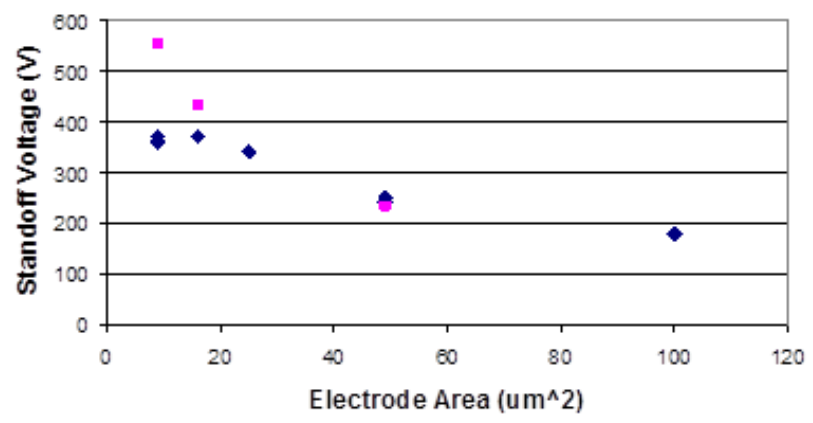

Figure 6: Single switch stand-off voltage vs. contact area, with simulated data shown as pink squares and experimental data in blue diamonds, showing good agreement until breakdown around $350 \mathrm{~V}$.
At first approximation, a contact with a gap in this range should have a standoff voltage of $<100 \mathrm{~V}$. In the literature [7], experiments have been run to better understand the modified Paschen curve where a curved surface (pin) is place in proximity with a flat plane. This configuration shows a low withstand voltage capability and has since been used as an upper limit to the typical MEMS switch design space. Experimental results of $300-350 \mathrm{~V}$ for $<1 \mu \mathrm{m}$ gaps determined that the configuration used for the modified Paschen curve did not apply to micro scale relays. This sustained voltage across the contacts is likely due to the gap being smaller than the mean free path of the gas molecules and thus minimizing the effect of avalanche breakdown by minimizing the ionization potential. At gaps smaller than the Paschen minimum of $327 \mathrm{~V}$ in air, field emission becomes significant. The contacts and their roughness have been designed and fabricated to minimize field emission effects at small gaps and have enabled sustained voltages $>300 \mathrm{~V}$ across a $1 \mu \mathrm{m}$ gap, a value that exceeds the limits of the modified Paschen curve

Switch designs capable of taking advantage of both the nickel alloy's structural robustness and conductivity as well as the small contact gap geometries that sustain a few hundred volts were fabricated into various device design configurations capable of handling kilowatts of power to efficiently transmitting RF signals in the $\mathrm{GHz}$ range. These designed leveraged the development of the nickel based alloy that serves as the electrically conductive spring element in the MEMS based (<100um) cantilever switch unit cell. The alloy's mechanical properties greatly expand the design space and enable high withstand voltage $(>250 \mathrm{~V})$ designs, a significant reduction in switch size $(\sim 5 \mathrm{X})$ compared to pure metals and improved lifetime and reliability of the device.

The process flow utilizes surface micromachining and uses the substrate as a carrier for the fabrication processing. Because the switch is surface micromachined with a maximum processing temperature of $300^{\circ}$ this process is transferable to different substrate materials. High power devices $(>1 \mathrm{~kW})$ are fabricated on silicon substrates for improved thermal conduction, while RF devices are fabricated on quartz or fused silica substrates for best in class RF isolation of better than -35 dB @ $6 \mathrm{GHz}$ by minimizing RF capacitive coupling through the substrate. The processing techniques used include lithography, physical vapor deposition (PVD) methods (electron beam evaporation and DC Magnetron sputtering), plasma enhanced chemical vapor deposition (PECVD), reactive ion etching (RIE), wet chemical etching, electroplating and other cleanroom based fabrication techniques.

The basic unit cell switch element is a free standing 50 $\mu \mathrm{m}$ wide cantilever beam that is anchored on one end, and that extends $\sim 50 \mu \mathrm{m}$ towards and $1 \mu \mathrm{m}$ above its separated contact on its opposite end. The basic switching element is fabricated using the Ni-alloy and each switching unit is copied multiple times on a die and arrayed in parallel to enhance a die's ability to carry current as needed. The array of beams simultaneously deflects approximately $1 \mu \mathrm{m}$ after it has been electrostatically actuated to make Ohmic contact between an upper and lower contact. When the switches are closed, they have a resistance of about $1 \Omega$ per unit cell switch element and a parallel array of hundreds of switches on a small die is capable of carrying $5 \mathrm{~A}$ of continuous current with very low on-state losses. Arc free mechanical switching is critical for switching power and has been achieved through a combination of fast switching speed and the ability to open the contacts at a forced and momentary artificially induced zero voltage. An example of the how the 


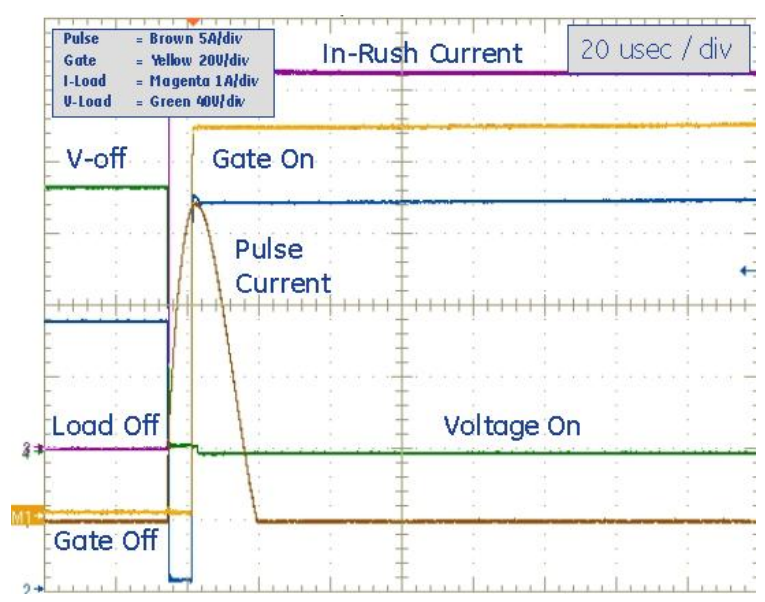

Figure 7: An oscilloscope trace capturing the switch turn on event (Gate Off to Gate On), at the microsecond scale, where the initial load current (Load Off to In-Rush Current) raises to 5 amperes on the resistive load.

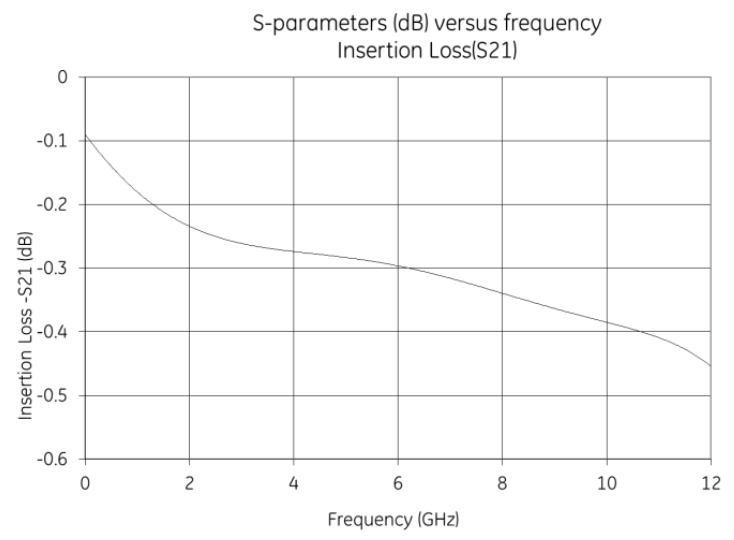

Figure 8: Insertion loss measurement of a 50-ohm impedance matched single unit cell RF MEMS switch showing better than $0.3 \mathrm{~dB}$ of loss at frequencies below $6 \mathrm{GHz}$.

switch and its circuitry closes into and turns on a 500W load is captured in the scope trace of figure 7.

In addition to low frequency (DC and AC) device and array configurations for the unit cell switch, 50-ohm impedance matched single unit cell RF MEMS relays were designed and fabricated on fused silica substrates. The fused silica enabled better than $35 \mathrm{~dB}$ of isolation and minimal on state insertion losses better than $0.3 \mathrm{~dB}$ on a given channel as measured in figure 8. Radio frequency tests are typically performed by a Vector Network Analyzer (VNA) that is capable of sweeping the frequency of interest. Electrical connections of MEMS switches are made with $50-\Omega$ GSG probes for VNA ports.

\section{CONCLUSIONS}

Newly engineered micromechanical structural materials will be required to enable a novel class of MEMS devices that go beyond the signal transmission and sensing capabilities of present commercial sensors and initiate a new class of MEMS based devices capable of manipulating kilowatts of power. This new class of devices requires nanostructured materials with the mechanical properties near that of silicon along with the conductivity of noble metals. We have developed an electroplated nanostructured Ni-alloy that has the conductivity of Nickel and a yield strength $>1 \mathrm{GPa}$ and demonstrated its device practicality by fabricating both die capable of transmitting and switching more than 1 kilowatts of power and die capable of low loss $(<0.3 \mathrm{~dB})$ and high isolation $(>35 \mathrm{~dB}) \mathrm{RF}$ MEMS relays.. The minimal strain rate of the nanostructured Ni-alloy makes possible the material resistance to stress induced deformation and enables a device to repeatedly operate for years at temperature. The combination of fast switching speed and broadband (DC to RF) signal operation along with the ability to control amperes of current and sustain hundreds of volts across micron sized air gaps has enabled the miniaturization of the mechanical relay to the chip scale for wide ranging applications in industrial sectors for power control to consumer communication driven applications requiring $\mathrm{RF}$ relays.

\section{ACKNOWLEDGEMENTS}

The authors would like to acknowledge the many people at GE Global Research who have had a hand in enabling all aspects of this technology from materials development through fabrication and into device test and integration; specifically Glenn Claydon for years of help and expertise in device layout and electrical test, Don Lipkin for his expertise and guidance in materials development, Sairam Sundaram and Sia Sarva for the work on lifing analysis and Steven Go, Robert Butler, Yongjae Lee and Joseph Iannotti for their guidance and help with RF design and characterization.

\section{REFERENCES}

[1] Rebeiz, Gabriel M., "RF MEMS Theory, Design, and Technology", Wiley, Hoboken, NJ 2003

[2] J. Wong, J. Lang, M. Schmidt, "An electrostaticallyactuated MEMS switch for power applications", Proceedings of the IEEE MEMS 2000 Conference, Miyazaki, 2000, pp. 633

[3] Majumder, S.; McGruer, N.E.; Adams, G.G.; , "Adhesion and contact resistance in an electrostatic MEMS micro switch," Micro Electro Mechanical Systems, 18th IEEE International Conference, 215- 218, 2005

[4] Hyman, D. and Mehregany, M., "Contact physics of gold microcontacts for MEMS", in Electrical Contacts, 1998.Proceedings of the Forty-Fourth IEEE Holm Conference. 133-140, 1998

[5] Townsend, J.S., "The Theory of Ionization of Gases by Collision", London: Constable \& Co. Ltd.1910

[6] Paschen, F., "Ueber die xum Funkenubergang in Luft, Wasserstoff und Kohlensaure bei verschiedenen Druckenerforderliche Potentialdifferenz", Annalen der Physik, .37, 69-96, 1889

[7] Slade, P., Taylor, E., "Electrical Breakdown in atmospheric air between closely spaced (0.2um-40um) electrical contacts", IEEE Transactions on Componenents and Packaging Technologies, vol. 25, pp 390-396, 2001

\section{CONTACT}

C. Keimel, tel: +1-518-387-7893; keimel@ research.ge.com 